\title{
Perioperative Blood Transfusion is Associated with Postoperative Systemic Inflammatory Response and Poorer Outcomes Following Surgery for Colorectal Cancer
}

\author{
Stephen T. McSorley, PhD, MRCS ${ }^{1}$ (1) , Alexander Tham ${ }^{1}$, Ross D. Dolan, MBChB, MRCS ${ }^{1}$, \\ Colin W. Steele, PhD, FRCS ${ }^{1}$, Jason Ramsingh, MSc, FRCS ${ }^{1}$, Campbell Roxburgh, PhD, FRCS ${ }^{1}$, \\ Paul G. Horgan, PhD, FRCS ${ }^{1}$, and Donald C. McMillan, PhD ${ }^{1}$ \\ Academic Unit of Surgery, University of Glasgow, Glasgow, UK
}

\begin{abstract}
Background. The present study investigated relationships between perioperative blood transfusion, postoperative systemic inflammatory response, and outcomes following surgery for colorectal cancer.

Methods. Data were recorded for patients $(n=544)$ undergoing potentially curative, elective surgery for colorectal cancer at a single center between 2012 and 2017. Transfusion history was obtained retrospectively from electronic records. Associations between blood transfusion, postoperative C-reactive protein (CRP), albumin, hemoglobin, complications, cancer-specific survival and overall survival (OS) were assessed using propensity score matching $(n=116)$.
\end{abstract}

Results. Of 544 patients, the majority were male ( $n=294$, $54 \%)$, over 65 years of age $(n=350,64 \%)$, and with colonic $(n=347,64 \%)$ node-negative disease $(n=353$, $65 \%)$. Eighty-six patients $(16 \%)$ required perioperative blood transfusion. In the unmatched cohort, blood transfusion was associated with higher median postoperative day (POD) 3 CRP $\{143$ [interquartile range (IQR) 96-221 mg/L] vs. 120 (IQR 72-188 mg/L); $p=0.004\}$, lower median POD 3 albumin [24 (IQR 20-26 g/L) vs. 27 (IQR 24-30 g/L); $p<0.001]$, more postoperative complications [odds ratio (OR) $3.28,95 \%$ confidence interval (CI) 2.03-5.29] and poorer OS [hazard ratio (HR) 3.18, 95\% CI 2.08-4.84]. In the propensity score matched cohort, blood

(C) The Author(s) 2019

First Received: 22 August 2019; Published Online: 29 October 2019

S. T. McSorley, PhD, MRCS

e-mail: s.mcsorley@doctors.org.uk transfusion was similarly associated with higher median POD 3 CRP [130 (IQR 93-196 mg/L) vs. 113 (IQR $66-173 \mathrm{mg} / \mathrm{L}) ; p=0.046]$, lower median POD 3 albumin [24 (IQR 20-26 g/L) vs. 26 (IQR 24-30 g/L); $p<0.001$, more postoperative complications (OR 2.91, 95\% CI 1.36-6.20) and poorer OS (HR 2.38, 95\% CI 0.99-5.73). Conclusions. Perioperative blood transfusion was associated with postoperative inflammation, complications, and poorer survival in patients undergoing colorectal cancer surgery, with and without propensity score techniques.

A significant proportion of patients undergoing surgery for colorectal cancer require allogeneic blood transfusion in the perioperative period. ${ }^{1}$ Such transfusions are associated with infective postoperative complications and anastomotic leak. ${ }^{2,3}$ They are also associated with disease recurrence, ${ }^{3,4}$ and this effect is even greater in the presence of infective complications. ${ }^{5}$ Therefore, it has long been hypothesized that allogeneic blood transfusion might impair the host adaptive immune response to both pathogens and circulating or micrometastatic tumor cells. ${ }^{6}$

There is increasing evidence that an exaggerated postoperative systemic inflammatory response following surgery for colorectal cancer is associated with postoperative complications and long-term survival. ${ }^{7}$ Furthermore, there is some observational evidence that modulation of this response can improve both short- ${ }^{8}$, and long-term outcomes. ${ }^{9}$ It may be that perioperative blood transfusion and postoperative complications have a negative impact on oncologic outcomes via a common pathway, the systemic inflammatory response. ${ }^{10}$ 
The aim of this study was to examine the association between perioperative blood transfusion and the magnitude of postoperative systemic inflammatory response, in particular C-reactive protein (CRP) and albumin, which have been reported to be reliable indicators of the magnitude of surgical injury. ${ }^{11}$ Furthermore, the study aimed to examine the impact of blood transfusion on the development of postoperative complications and long-term outcomes following surgery for colorectal cancer.

\section{MATERIALS AND METHODS}

\section{Patients}

This observational study was a retrospective analysis of a prospectively collected and maintained cohort. Inclusion criteria were patients from a single surgical unit with colorectal cancer who underwent surgery with curative intent between January 2012 and June 2017. Exclusion criteria were emergency surgery, palliative presentation, pre-existing systemic inflammatory comorbidity, hematological malignancy, and long-term steroids.

Prior to and following surgery, all cases were discussed at a multidisciplinary colorectal oncology meeting. Antimicrobial and venous thromboembolism prophylaxis was administered at the time of surgery. Perioperative care was standardized using an 'enhanced recovery' protocol. A proportion of patients received postoperative nausea and vomiting prophylaxis in the form of intravenous dexamethasone during surgery, at the discretion of the consultant anesthetist. ${ }^{8,12}$

During the study period, there was no formal perioperative blood transfusion protocol. As reported previously, ${ }^{13}$ between June 2016 and June 2017 a preoperative iron replacement protocol was in place for the relatively small number of patients found to have true iron-deficient anemia.

On each day from surgery to discharge, patients had a bedside review by the responsible operating team, and blood samples were drawn for CRP and albumin. The patient's surgical team arranged further investigation, and/ or intervention, based on clinical assessment and these blood results.

\section{METHODS}

Patient data were recorded securely and anonymized prior to analysis. The data collected included factors previously reported to be associated with the postoperative systemic inflammatory response, including modified Glasgow Prognostic Score (mGPS), laparoscopic resection, body mass index (BMI) and American Society of
Anesthesiologists (ASA) score. ${ }^{14}$ TNM stage (TNM, 5th ed American Joint Committee on Cancer [AJCC]) and pathological features were recorded, as were demographic variables.

Hematological parameters were recorded from full blood count samples taken within 2 weeks prior to surgery. Patients were classified as having anemia based on World Health Organization (WHO) guidelines for males, i.e. hemoglobin $(\mathrm{Hb})<130 \mathrm{mg} / \mathrm{L}$, and females, i.e. $\mathrm{Hb}<$ $120 \mathrm{mg} / \mathrm{L} .{ }^{15}$ Furthermore, anemic patients were classified as having microcytic anemia with mean corpuscular volume $(\mathrm{MCV})<80 \mathrm{f} / \mathrm{L}$, normocytic anemia with $\mathrm{MCV}$ $80-100 \mathrm{f} / \mathrm{L}$, or macrocytic anemia with MCV $>100 \mathrm{f} / \mathrm{L}$.

Retrospective linkage with the blood transfusion electronic database allowed recording of the transfusion of packed red cells (PRCs), number of units transfused, and the time given. Perioperative blood transfusion was defined as any transfusion of PRCs from 30 days prior to 30 days following surgery. All units of PRCs were leucodepleted following screening for blood-borne viruses (HIV, Human T-lymphotropic virus, and hepatitis $\mathrm{B}$ and $\mathrm{C}$ ), malaria, and syphilis. All PRCs were stored between 2 and $6{ }^{\circ} \mathrm{C}$ and used within 35 days of donation.

Serum concentrations of CRP $(\mathrm{mg} / \mathrm{L})$ were measured using an autoanalyzer (Architect; Abbot Diagnostics, Maidenhead, UK) with a lower detectable limit of $0.2 \mathrm{mg} /$ $\mathrm{L}$, as was serum albumin (normal range $35-50 \mathrm{~g} / \mathrm{L}$ ). The mGPS was calculated in patients for whom preoperative serum CRP and albumin were available. ${ }^{16}$ On days 3 and 4 , after surgery for those patients with available CRP and albumin, the validated postoperative Glasgow Prognostic Score (poGPS) was calculated. ${ }^{7}$

If complications occurred between surgery and first clinic follow-up, they were recorded according to the most severe, using the Clavien-Dindo grade, ${ }^{17}$ and type. Infective complications were categorized as described previously. ${ }^{18}$ Superficial surgical site infection was defined as the presence of pus either drained from the wound or discharging spontaneously. Deep surgical site infection was diagnosed either on imaging, radiological drainage, or surgical drainage of intra-abdominal pus, while anastomotic leak was diagnosed at laparotomy or on crosssectional imaging. Lower respiratory tract infection was defined as consolidation on chest radiograph or computed tomography (CT) scan associated with pyrexia and requiring antibiotic treatment, whereas septicemia was defined as a combination of the systemic inflammatory response syndrome and positive blood culture. Urinary tract- or catheter-related infection was only diagnosed in the presence of the systemic inflammatory response syndrome and positive urine culture.

The study was approved by the West of Scotland Research Ethics Committee, Glasgow. 


\section{Statistical Analysis}

In the unmatched cohort, categorical data were compared using the Chi square test and Chi square for linear association where appropriate. Postoperative CRP, albumin, and $\mathrm{Hb}$ were non-normal, presented as medians and ranges, and compared using the Mann-Whitney $U$ test. The magnitude of CRP, albumin, and $\mathrm{Hb}$ by each postoperative day (POD) was displayed graphically as $95 \%$ confidence intervals of the median.

Following exclusion of deaths within 30 days of surgery or during the index admission, cancer-specific survival (CSS) was defined as the time from the day of resection to the day of colorectal cancer death. Overall survival (OS) was defined as the time from the day of resection to that of all-cause death. Survival was presented as a cumulative proportion of patients alive at 5 years following surgery and assessed using the log-rank test.

Multivariate logistic regression generated propensity scores, predicting the probability of having received a perioperative transfusion, based on variables associated with the postoperative systemic inflammatory response or complications $^{14}$; age, sex, BMI, ASA, mGPS, tumor site, TNM stage, neojuvant chemoradiotherapy, surgical approach (open or laparoscopic), administration of intraoperative dexamethasone, and preoperative anemia. Patients who received a perioperative blood transfusion were then matched 1:1 with a patient who did not, using the closest propensity score on the logit scale (calliper $<0.05$, order of match selection randomized, no replacement). Categorical data were compared using McNemar's test, while continuous data were compared using the related samples Wilcoxon signed-rank test. The appropriateness of the propensity score matching was assessed visually by the frequency of propensity scores in each group before and after matching.

Sensitivity analyses compared the effect size of perioperative blood transfusion in relation to CRP concentrations on postoperative day 3 using linear regression, postoperative complications using binary logistic regression presented as odd ratios (OR) and 95\% CI, and overall survival using Cox regression presented as hazard ratios (HR) and 95\% CI, between the unmatched cohort, propensity score matched cohort, and using propensity score regression.

Factors associated with postoperative complications and OS among patients receiving perioperative blood transfusion were examined using multivariate binary logistic regression and multivariate Cox regression, respectively. Variables associated with outcomes at a univariate level of $p<0.1$ were entered into a backward conditional model, where $p<0.05$ was considered statistically significant.
In all tests, a two-sided $p$ value $<0.05$ was considered statistically significant. Propensity scoring matching and all statistical analyses were performed using IBM SPSS version 24 for Windows (IBM Corporation, Armonk, NY, USA).

\section{RESULTS}

\section{Patients}

Overall, 544 patients were included, the majority of whom were male $(n=294,54 \%)$, over 65 years of age $(n=350,64 \%)$, and with colonic $(n=347,64 \%)$ and nodenegative disease $(n=353,65 \%)$. One hundred and ninety patients $(35 \%)$ were anemic prior to surgery, of whom 42 (8\%) had microcytic anemia and 148 (27\%) had normocytic anemia. Eighty-six patients $(16 \%)$ required allogeneic blood transfusion within the perioperative period, with 9 patients $(2 \%)$ receiving a preoperative blood transfusion, $67(12 \%)$ receiving an intraoperative or postoperative blood transfusion, and $10(2 \%)$ receiving a transfusion at multiple time points. Two hundred and seven patients (38\%) experienced a complication, of which $130(24 \%)$ were infective, with $23(4 \%)$ anastomotic leaks, and 53 (10\%) were Clavien-Dindo grade 3-5. There were $7(1 \%)$ deaths within 30 days of surgery. During the follow-up period, 87 patients died (16\%), $52(10 \%)$ cases of which were due to colorectal cancer. The median follow-up of those alive at the time of censoring was 43 months (range 12-77).

\section{Perioperative Blood Transfusion in the Unmatched Cohort}

In the unmatched cohort (Table 1), perioperative blood transfusion was associated with increasing age $(p=0.038)$, lower BMI $(p=0.028)$, higher ASA grade $(p=0.001)$, mGPS of $2(p<0.001)$, open surgery $(p<0.001)$, and non-administration of dexamethasone $(p<0.001)$. Patients who received a perioperative blood transfusion had a significantly lower preoperative $\mathrm{Hb}(p<0.001)$, and a significantly higher proportion had normocytic anemia $(p<0.001)$.

Patients who received a perioperative blood transfusion had a significantly lower $\mathrm{Hb}$ on PODs 1-5 (Fig. 1a), a significantly higher CRP on PODs 1-3 (Fig. 1c), and a significantly lower albumin on PODs 1-5 (Fig. 1e). A higher proportion of patients who received a perioperative blood transfusion had a poGPS of 2 on PODs 3 (32\% vs. $15 \% ; p=0.004)$ and $4(29 \%$ vs. $12 \% ; p=0.010)$.

Patients who received a perioperative blood transfusion had a significantly higher rate of postoperative 
TABLE 1 Clinicopathological characteristics of patients undergoing elective, open surgery for colorectal cancer receiving any perioperative blood transfusion-unmatched cohort

\begin{tabular}{|c|c|c|c|c|}
\hline Characteristics & All $[n=544]$ & No transfusion $[n=458]$ & Transfusion $[n=86]$ & $p$ value \\
\hline \multicolumn{5}{|l|}{ Clinicopathological } \\
\hline Age, years $(<65 / 65-74 / \geq 75)$ & $194 / 208 / 142$ & $168 / 180 / 110$ & $26 / 28 / 32$ & 0.038 \\
\hline Sex (male/female) & $294 / 248$ & $253 / 203$ & $41 / 45$ & 0.196 \\
\hline BMI $\left(<20 / 20-25 / 26-30 />30 \mathrm{~kg} / \mathrm{m}^{2}\right)$ & $33 / 169 / 168 / 164$ & $23 / 142 / 141 / 145$ & $10 / 27 / 27 / 19$ & 0.028 \\
\hline ASA $(1 / 2 / 3 / 4)$ & $131 / 234 / 149 / 14$ & $117 / 204 / 119 / 8$ & $14 / 30 / 30 / 6$ & 0.001 \\
\hline Iron infusion (yes/no) & $19 / 531$ & $18 / 440$ & $1 / 85$ & 0.336 \\
\hline Approach (laparoscopic/open) & $269 / 263$ & $246 / 200$ & $23 / 63$ & $<0.001$ \\
\hline Perioperative dexamethasone (yes/no) & $334 / 138$ & $302 / 104$ & $32 / 34$ & $<0.001$ \\
\hline Tumor site (colon/rectum) & $347 / 196$ & $285 / 173$ & $62 / 23$ & 0.065 \\
\hline \multicolumn{5}{|l|}{ Operation duration, mins [median $(I Q R)]$} \\
\hline Colonic & $180(131-252)$ & $181(135-240)$ & $173(120-207)$ & 0.107 \\
\hline Rectal & $275(210-335)$ & $275(209-335)$ & $283(228-409)$ & 0.811 \\
\hline TNM stage (0/I/II/III) & $13 / 127 / 213 / 183$ & $10 / 117 / 174 / 152$ & $3 / 10 / 39 / 31$ & 0.132 \\
\hline Neoadjuvant treatment (yes/no) & $78 / 458$ & $62 / 391$ & $16 / 67$ & 0.180 \\
\hline \multicolumn{5}{|l|}{ Hematological } \\
\hline Preoperative $\mathrm{Hb}, \mathrm{g} / \mathrm{L}$ [median (IQR)] & $131(118-144)$ & $133(124-146)$ & $106(98-121)$ & $<0.001$ \\
\hline Preoperative anemia (no/microcytic/normocytic) ${ }^{a}$ & $354 / 42 / 148$ & $333 / 20 / 105$ & $21 / 22 / 43$ & $<0.001$ \\
\hline POD $1 \mathrm{Hb}, \mathrm{g} / \mathrm{L}$ [median (IQR)] & $110(98-123)$ & $113(103-125)$ & $94(87-101)$ & $<0.001$ \\
\hline POD $2 \mathrm{Hb}, \mathrm{g} / \mathrm{L}$ [median(IQR)] & $107(97-120)$ & $110(100-123)$ & $90(82-101)$ & $<0.001$ \\
\hline POD $3 \mathrm{Hb}, \mathrm{g} / \mathrm{L}$ [median (IQR)] & $108(98-121)$ & $111(101-124)$ & $95(90-101)$ & $<0.001$ \\
\hline POD $4 \mathrm{Hb}, \mathrm{g} / \mathrm{L}$ [median (IQR)] & $110(99-122)$ & $114(103-125)$ & $96(92-105)$ & $<0.001$ \\
\hline POD $5 \mathrm{Hb}, \mathrm{g} / \mathrm{L}$ [median (IQR)] & $111(99-125)$ & $114(103-127)$ & $98(92-107)$ & $<0.001$ \\
\hline \multicolumn{5}{|l|}{ Systemic inflammation } \\
\hline Preoperative mGPS $(0 / 1 / 2)^{\mathrm{b}}$ & $399 / 42 / 56$ & $345 / 37 / 37$ & $54 / 5 / 19$ & $<0.001$ \\
\hline POD 1 CRP, mg/L [median (IQR)] & $83(55-116)$ & $80(52-112)$ & $101(72-138)$ & $<0.001$ \\
\hline POD 2 CRP, mg/L [median (IQR)] & $132(82-197)$ & $127(79-191)$ & $164(117-225)$ & $<0.001$ \\
\hline POD 3 CRP, mg/L [median (IQR)] & $127(76-192)$ & $120(72-188)$ & $143(96-221)$ & 0.004 \\
\hline POD 4 CRP, mg/L [median (IQR)] & $101(64-172)$ & $100(63-167)$ & $115(72-239)$ & 0.050 \\
\hline POD 5 CRP, mg/L [median (IQR)] & $86(51-158)$ & $83(49-149)$ & $107(55-232)$ & 0.051 \\
\hline POD 1 albumin, g/L [median (IQR)] & $28(25-31)$ & $28(25-31)$ & $24(21-26)$ & $<0.001$ \\
\hline POD 2 albumin, g/L [median (IQR)] & $27(24-31)$ & $28(25-31)$ & $23(21-26)$ & $<0.001$ \\
\hline POD 3 albumin, g/L [median (IQR)] & $26(24-30)$ & $27(24-30)$ & $24(20-26)$ & $<0.001$ \\
\hline POD 4 albumin, g/L [median (IQR)] & $26(23-29)$ & $27(24-30)$ & $23(20-26)$ & $<0.001$ \\
\hline POD 5 albumin, g/L [median (IQR)] & $26(23-29)$ & $27(24-30)$ & $23(19-25)$ & $<0.001$ \\
\hline poGPS $3(0 / 1 / 2)^{\mathrm{c}}$ & $304 / 115 / 88$ & $262 / 102 / 62$ & $42 / 13 / 26$ & 0.004 \\
\hline poGPS $4(0 / 1 / 2)^{\mathrm{c}}$ & $300 / 62 / 64$ & $249 / 58 / 41$ & $51 / 4 / 23$ & 0.010 \\
\hline \multicolumn{5}{|l|}{ Short-term outcomes } \\
\hline Any complication (yes/no) & $207 / 332$ & $154 / 301$ & $53 / 31$ & $<0.001$ \\
\hline Infective complication (yes/no) & $130 / 409$ & $91 / 364$ & $39 / 45$ & $<0.001$ \\
\hline Anastomotic leak (yes/no) & $23 / 516$ & $8 / 447$ & $15 / 69$ & $<0.001$ \\
\hline Clavien-Dindo grade $(0-2 / 3-5)$ & $484 / 53$ & $429 / 24$ & $55 / 29$ & $<0.001$ \\
\hline 30-day mortality (yes/no) & $7 / 532$ & $5 / 449$ & $1 / 83$ & 0.451 \\
\hline Length of stay, days [median (IQR)] & $7(5-12)$ & $7(5-10)$ & $12(7-19)$ & $<0.001$ \\
\hline \multicolumn{5}{|l|}{ Long-term outcomes } \\
\hline Adjuvant treatment (yes/no) & $165 / 295$ & $146 / 240$ & $19 / 55$ & 0.048 \\
\hline 5-year CSS (\%) & 86 & 89 & 68 & $<0.001$ \\
\hline
\end{tabular}


TABLE 1 continued

\begin{tabular}{lllll}
\hline Characteristics & All $[n=544]$ & No transfusion $[n=458]$ & Transfusion $[n=86]$ & $p$ value \\
\hline 5-year OS $(\%)$ & 79 & 82 & 59 & $<0.001$ \\
\hline
\end{tabular}

Data are expressed as $n$ unless otherwise specified

ASA American Society of Anesthesiologists grade, BMI body mass index, $H b$ Hemoglobin, CRP C-reactive protein, $O S$ overall survival, CSS cancer-specific survival, $P O D$ postoperative day, $I Q R$ interquartile range, $m G P S$ modified Glasgow Prognostic Score, poGPS postoperative Glasgow Prognostic Score, $M C V$ mean corpuscular volume

${ }^{\mathrm{a}}$ Males $=\mathrm{Hb}<130 \mathrm{~g} / \mathrm{L}$, females $=\mathrm{Hb}<120 \mathrm{~g} / \mathrm{L} ;$ microcytic $=$ anemia and $\mathrm{MCV}<80 \mathrm{fL}$; normocytic $=$ anemia and $\mathrm{MCV} 80-100 \mathrm{fL}$

${ }^{\mathrm{b}} 0=\mathrm{CRP}<10 \mathrm{mg} / \mathrm{L} ; 1=\mathrm{CRP} \geq 10 \mathrm{mg} / \mathrm{L}$ and albumin $\geq 35 \mathrm{~g} / \mathrm{L} ; 2=\mathrm{CRP} \geq 10 \mathrm{mg} / \mathrm{L}$ and albumin $<35 \mathrm{~g} / \mathrm{L}$

${ }^{\mathrm{c}} 0=\mathrm{CRP}<150 \mathrm{mg} / \mathrm{L} ; 1=\mathrm{CRP} \geq 150 \mathrm{mg} / \mathrm{L}$ and albumin $\geq 25 \mathrm{~g} / \mathrm{L} ; 2=\mathrm{CRP} \geq 150 \mathrm{mg} / \mathrm{L}$ and albumin $<25 \mathrm{~g} / \mathrm{L}$

complications $(63 \%$ vs. $34 \% ; p<0.001)$, infective complications $(46 \%$ vs. $20 \% ; p<0.001)$, anastomotic leak (18\% vs. $2 \% ; p<0.001)$, and Clavien-Dindo grade $3-5$ complications ( $35 \%$ vs. $5 \% ; p<0.001$ ), as well as a longer median length of stay (12 vs. 7 days; $p<0.001$ ).

Perioperative blood transfusion was significantly associated with poorer 5 -year CSS $(68 \%$ vs. $89 \% ; p<0.001)$ and poorer 5 -year OS $(59 \%$ vs. $82 \%$; $p<0.001)$.

\section{Perioperative Blood Transfusion in the Propensity Score Matched Cohort}

Propensity scores could not be assigned to 121 patients due to missing covariate data, leaving 423 patients with propensity scores, of whom 62 had received a perioperative blood transfusion and 361 had not (Fig. 2a). One hundred and sixteen patients were matched (58 in each group) based on their propensity scores, with a subsequent improvement in the balance of their distribution (Fig. 2b).

In the propensity score matched cohort (Table 2), patients receiving a perioperative blood transfusion had significantly lower $\mathrm{Hb}$ on POD 2, but at no other time point (Fig. 1b). Patients receiving a perioperative blood transfusion had a significantly higher CRP on PODs 1-4 (Fig. 1d), and significantly lower albumin on PODs 1-5 (Fig. 1f). A higher proportion of patients who received a perioperative blood transfusion had a poGPS of 2 on POD 3 ( $28 \%$ vs. $13 \% ; p=0.031$ ), but not on POD 4 ( $p=0.344$ ).

Patients who received a perioperative blood transfusion had a significantly higher rate of postoperative complications $(59 \%$ vs. $33 \% ; p=0.017)$, anastomotic leak ( $15 \%$ vs. $2 \% ; p=0.021$ ), and Clavien-Dindo grade 3-5 complications ( $34 \%$ vs. $5 \% ; p<0.001)$, as well as a longer median length of stay ( 11 vs. 7 days; $p=0.011$ ).

There was no significant difference in 5-year CSS (\%) or 5 -year OS (\%) between the propensity score matched groups.

\section{Sensitivity Analyses}

A similarly statistically significant association was found at linear regression with CRP as the dependent variable and blood transfusion as the explanatory variable, in the unmatched cohort, when patients with major complications (Clavien-Dindo grades 3-5) were excluded from the analysis, when using propensity score regression, and in the propensity score matched cohort (Table 3 ). The same was found using binary logistic regression with postoperative complication as the outcome variable, and Cox regression with OS as the outcome variable.

\section{Factors Associated with Poorer Outcomes Among Patients Receiving Perioperative Blood Transfusion}

When those patients who received a perioperative blood transfusion $(n=86)$ were examined (Table 4$)$, at univariate binary logistic regression, surgery for rectal cancer $(p=0.050)$, and increasing day 3 poGPS $(p=0.007)$ were associated with postoperative complications. At multivariate analysis, only day 3 poGPS remained significantly associated with postoperative complications (OR 2.47, 95\% CI 1.93-3.15; $p<0.001)$.

At univariate Cox regression, increasing ASA $(p=0.001)$, T stage $(p=0.029)$, and mGPS $(p=0.007)$ were significantly associated with poorer OS. At multivariate analysis, ASA (HR 1.96, 95\% CI 1.16-3.33; $p=0.012$ ) and mGPS (HR 1.69, 95\% CI 1.08-2.62; $p=0.020$ ) remained independently associated with OS.

\section{DISCUSSION}

The results of the present study report a significant association between perioperative blood transfusion, an exaggerated postoperative systemic inflammatory response, postoperative complications and survival following surgery for colorectal cancer. This remained the case even after propensity score matching for variables 

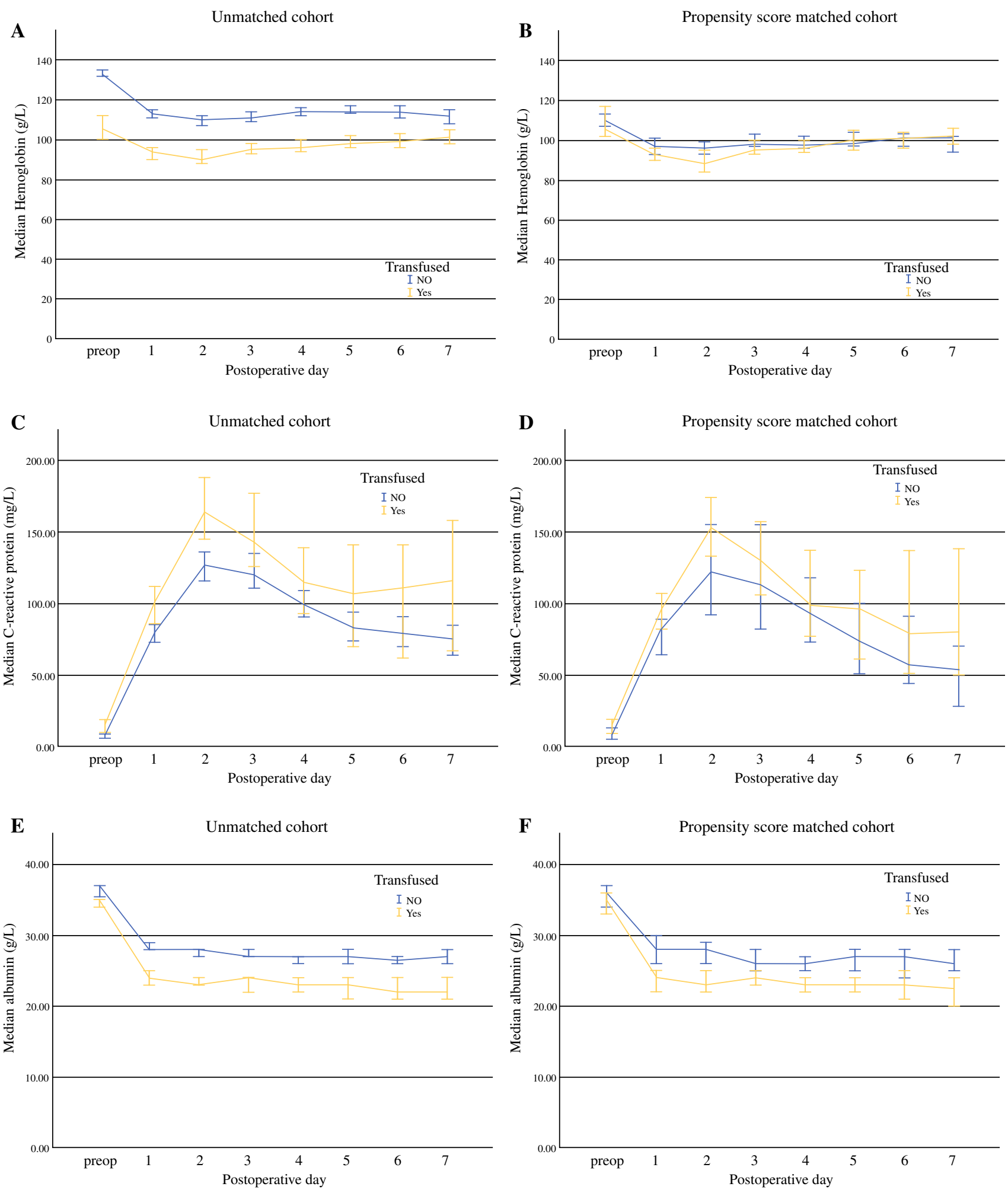

FIG. 1 Line charts comparing patients who did (yellow lines) and did not (blue lines) receive perioperative blood transfusions with regard to postoperative median hemoglobin concentrations $(\mathrm{g} / \mathrm{L})$ in the a unmatched and b propensity score matched cohorts, postoperative median CRP concentrations $(\mathrm{mg} / \mathrm{L})$ in the

c unmatched and d propensity score matched cohorts, and postoperative median albumin concentrations $(\mathrm{g} / \mathrm{L})$ in the e unmatched and $\mathbf{f}$ propensity score matched cohorts. Bars represent 95\% confidence intervals. preop preoperative, CRP C-reactive protein 
FIG. 2 Histograms of distribution of propensity scores among patients who did and did not receive perioperative blood transfusion in the a unmatched cohort and $\mathbf{b}$ propensity score matched cohort

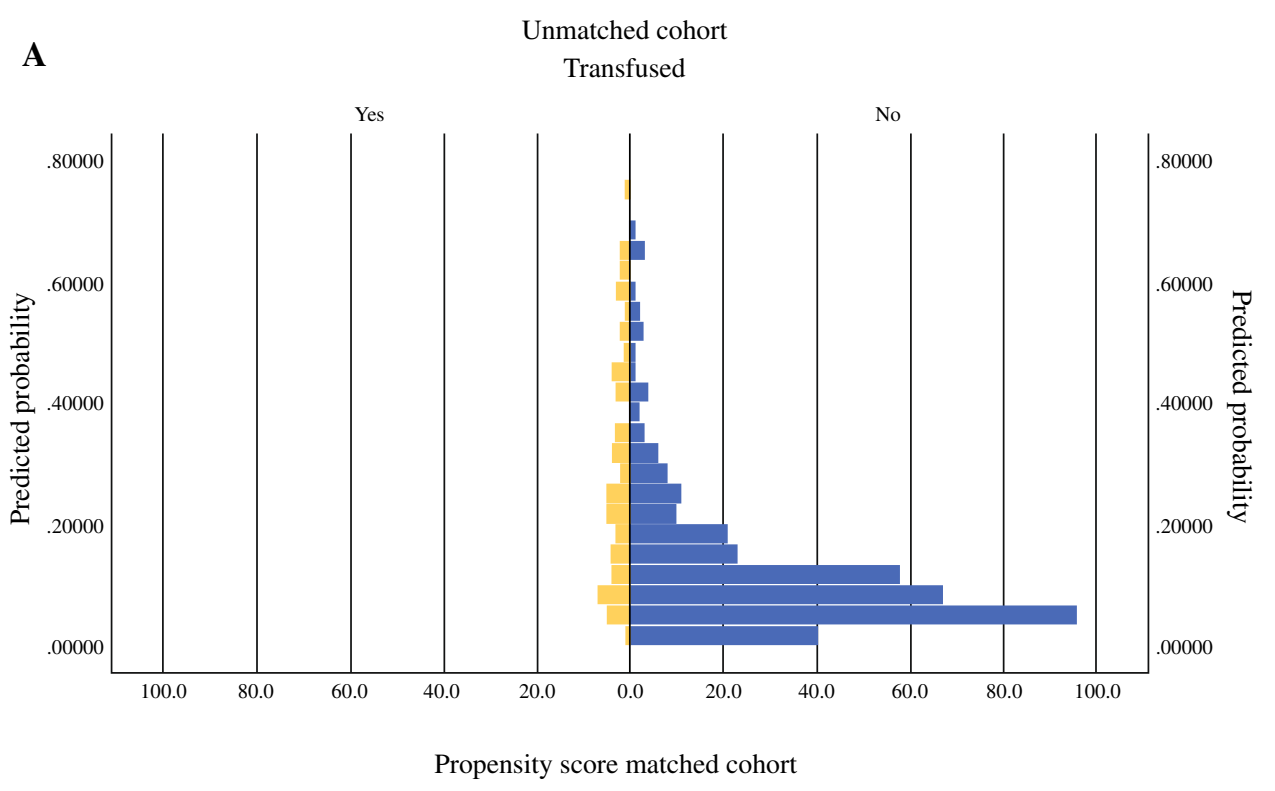

$\mathbf{B}$

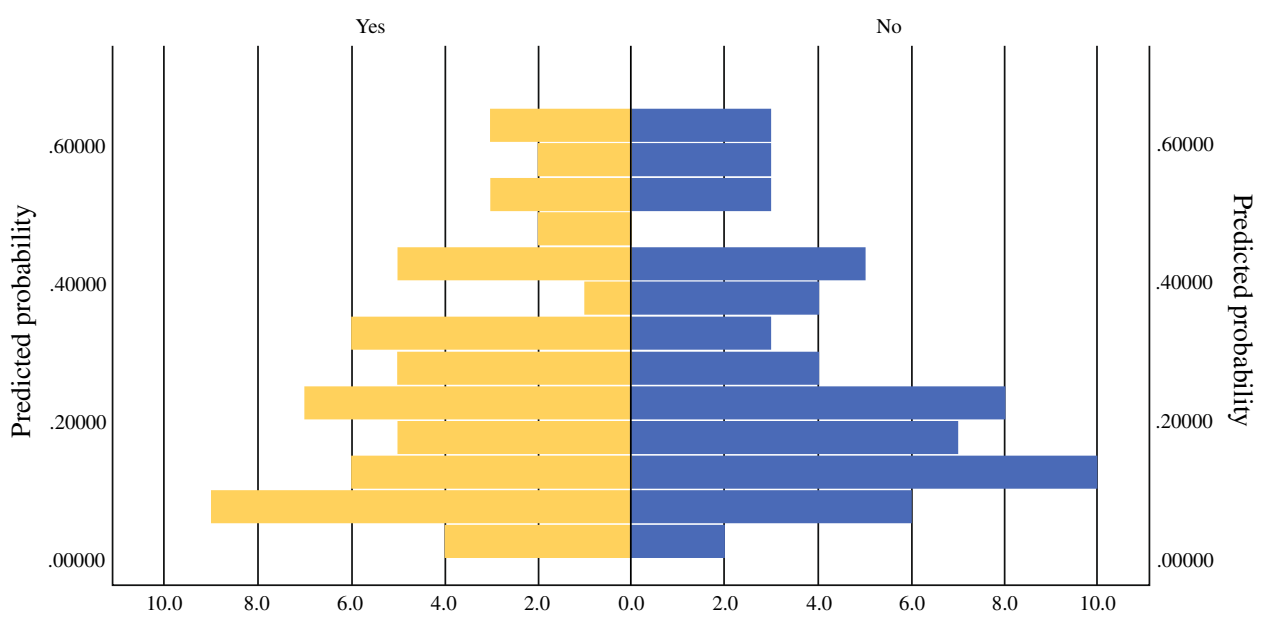

known to be associated with blood transfusion, postoperative systemic inflammatory response, and postoperative complications. Although blood transfusion is recognized to be associated with healthcare-related serious infection ${ }^{19}$ and short- and long-term outcomes following colorectal cancer resection, this study lends evidence to one of the proposed mechanisms-modulation of the host immune response to surgery.

There is increasing evidence that an exaggerated postoperative systemic inflammatory response is associated with postoperative complications and poorer oncologic outcomes in patients with gastrointestinal cancer. ${ }^{20-22}$ It is hypothesized that the primarily innate immune response to the trauma of surgery leads to relative suppression of adaptive responses. ${ }^{10,23}$ The reduced efficacy of this useful anticancer response, coupled with the pro-metastatic nature of the innate cellular, cytokine and neurohormonal surgical response, may increase the risk of recurrence. ${ }^{24}$ Furthermore, it has been observed that modulation of the postoperative systemic inflammatory response with perioperative corticosteroids is associated with fewer postoperative complications and improved survival; however, these findings are yet to be confirmed in prospective trials. $^{8,9}$

Allogeneic blood transfusion in the perioperative period is widely thought to be negatively associated with shortterm postoperative outcomes and survival in patients undergoing surgery for cancer, with an older Cochrane review, ${ }^{4}$ a recent large propensity score matched study of over 4000 patients, ${ }^{25}$ and a very recent comprehensive meta-analysis ${ }^{3}$ reporting this to be the case. However, two propensity score matched cohorts from a single group of researchers found no negative impact on survival in colon ${ }^{26}$ or rectal cancer, ${ }^{27}$ and a smaller recent observational study 
TABLE 2 Clinicopathological characteristics of patients undergoing elective, open surgery for colorectal cancer receiving any perioperative blood transfusion-propensity score matched cohort

\begin{tabular}{|c|c|c|c|c|}
\hline Characteristics & All $[n=116]$ & No transfusion $[n=58]$ & Transfusion $[n=58]$ & $p$ value \\
\hline \multicolumn{5}{|l|}{ Clinicopathological } \\
\hline Age, years $(<65 / 65-74 / \geq 75)$ & $35 / 39 / 42$ & $16 / 22 / 20$ & $19 / 17 / 22$ & - \\
\hline Sex (male/female) & $62 / 54$ & $32 / 26$ & $30 / 28$ & - \\
\hline BMI $\left(<20 / 20-25 / 26-30 />30 \mathrm{~kg} / \mathrm{m}^{2}\right)$ & $10 / 35 / 40 / 31$ & $5 / 20 / 17 / 16$ & $5 / 15 / 23 / 15$ & - \\
\hline ASA $(1 / 2 / 3 / 4)$ & $16 / 49 / 48 / 3$ & $7 / 26 / 24 / 1$ & $9 / 23 / 24 / 2$ & - \\
\hline Iron infusion (yes/no) & $8 / 108$ & $8 / 50$ & $0 / 58$ & - \\
\hline Approach (laparoscopic/open) & $44 / 72$ & $25 / 33$ & $19 / 39$ & - \\
\hline \multicolumn{5}{|l|}{ Operation duration, min [median (IQR)] } \\
\hline Colonic & $164(120-204)$ & $150(120-193)$ & $171(130-210)$ & - \\
\hline Rectal & $292(240-409)$ & $293(240-360)$ & $292(251-409)$ & - \\
\hline Perioperative dexamethasone (yes/no) & $72 / 44$ & $42 / 16$ & $30 / 28$ & - \\
\hline Tumor site (colon/rectum) & $89 / 27$ & $48 / 10$ & $41 / 17$ & - \\
\hline TNM stage $(0 / \mathrm{I} / \mathrm{II} / \mathrm{III})$ & $4 / 15 / 52 / 45$ & $1 / 8 / 26 / 23$ & $3 / 7 / 26 / 22$ & - \\
\hline Neoadjuvant treatment (yes/no) & $19 / 95$ & $9 / 48$ & $10 / 47$ & - \\
\hline \multicolumn{5}{|l|}{ Hematological } \\
\hline Preoperative $\mathrm{Hb}, \mathrm{g} / \mathrm{L}$ [median (IQR)] & $109(101-118)$ & $110(105-117)$ & $106(98-128)$ & - \\
\hline Preoperative anemia (no/microcytic/normocytic) ${ }^{a}$ & $20 / 28 / 64 / 4$ & $2 / 15 / 38 / 3$ & $18 / 13 / 26 / 1$ & - \\
\hline POD $1 \mathrm{Hb}, \mathrm{g} / \mathrm{L}$ [median (IQR)] & $95(88-103)$ & $97(91-104)$ & $93(86-100)$ & 0.063 \\
\hline POD $2 \mathrm{Hb}, \mathrm{g} / \mathrm{L}$ [median (IQR)] & $93(84-101)$ & $96(90-101)$ & $89(82-101)$ & 0.033 \\
\hline POD $3 \mathrm{Hb}, \mathrm{g} / \mathrm{L}$ [median (IQR)] & $96(90-104)$ & $98(89-105)$ & $95(90-103)$ & 0.443 \\
\hline POD $4 \mathrm{Hb}, \mathrm{g} / \mathrm{L}$ [median (IQR)] & $97(93-106)$ & $98(94-107)$ & $96(92-106)$ & 0.597 \\
\hline POD 5 Hb, g/L [median (IQR) & $99(93-107)$ & $99(95-108)$ & $100(92-107)$ & 0.820 \\
\hline \multicolumn{5}{|l|}{ Systemic inflammation } \\
\hline Preoperative mGPS $(0 / 1 / 2)^{\mathrm{b}}$ & $8 / 82 / 26$ & $43 / 4 / 11$ & $39 / 4 / 15$ & - \\
\hline POD 1 CRP, mg/L [median(IQR)] & $87(64-117)$ & $83(55-112)$ & $96(71-126)$ & 0.024 \\
\hline POD 2 CRP, mg/L [median(IQR)] & $143(86-198)$ & $122(78-183)$ & $153(117-201)$ & 0.011 \\
\hline POD 3 CRP, mg/L [median (IQR)] & $126(81-177)$ & $113(66-173)$ & $130(93-196)$ & 0.046 \\
\hline POD 4 CRP, mg/L [median (IQR)] & $97(59-174)$ & $93(50-157)$ & $99(63-239)$ & 0.029 \\
\hline POD 5 CRP, mg/L [median (IQR)] & $81(41-144)$ & $74(42-118)$ & $96(40-208)$ & 0.264 \\
\hline POD 1 albumin, g/L [median (IQR)] & $26(23-29)$ & $28(25-31)$ & $24(21-26)$ & $<0.001$ \\
\hline POD 2 albumin, g/L [median (IQR)] & $25(23-29)$ & $28(24-31)$ & $23(21-26)$ & $<0.001$ \\
\hline POD 3 albumin, g/L [median(IQR)] & $25(22-27)$ & $26(24-30)$ & $24(20-26)$ & $<0.001$ \\
\hline POD 4 albumin, g/L [median (IQR)] & $24(22-27)$ & $26(23-29)$ & $23(21-26)$ & 0.025 \\
\hline POD 5 albumin, g/L [median (IQR)] & $24(22-28)$ & $27(24-29)$ & $23(19-25)$ & 0.003 \\
\hline poGPS $3(0 / 1 / 2)^{\mathrm{c}}$ & $66 / 21 / 22$ & $35 / 13 / 7$ & $31 / 8 / 15$ & 0.031 \\
\hline poGPS $4(0 / 1 / / 2)^{\mathrm{c}}$ & $69 / 8 / 20$ & $32 / 6 / 7$ & $37 / 2 / 13$ & 0.344 \\
\hline \multicolumn{5}{|l|}{ Short-term outcomes } \\
\hline Any complication (yes/no) & $53 / 63$ & $19 / 39$ & $34 / 24$ & 0.017 \\
\hline Infective complication (yes/no) & $33 / 83$ & $11 / 47$ & $22 / 36$ & 0.061 \\
\hline Anastomotic leak (yes/no) & $10 / 106$ & $1 / 57$ & $9 / 49$ & 0.021 \\
\hline Clavien-Dindo grade $(0-2 / 3-5)$ & $23 / 93$ & $55 / 3$ & $38 / 20$ & $<0.001$ \\
\hline 30-day mortality (yes/no) & $1 / 115$ & $0 / 58$ & $1 / 57$ & - \\
\hline Length of stay, days [median (IQR)] & $8(6-13)$ & $7(5-12)$ & $11(6-16)$ & 0.011 \\
\hline \multicolumn{5}{|l|}{ Long-term outcomes } \\
\hline Adjuvant treatment (yes/no) & $32 / 63$ & $19 / 28$ & $13 / 35$ & 0.732 \\
\hline 5-year CSS (\%) & 78 & 90 & 70 & 0.199 \\
\hline
\end{tabular}


TABLE 2 continued

\begin{tabular}{lllll}
\hline Characteristics & All $[n=116]$ & No transfusion $[n=58]$ & Transfusion $[n=58]$ & $p$ value \\
\hline 5-year OS $(\%)$ & 72 & 85 & 63 & 0.094 \\
\hline
\end{tabular}

Data are expressed as $n$ unless otherwise specified

ASA American Society of Anesthesiologists grade, BMI body mass index, $H b$ hemoglobin, $C R P$ C-reactive protein, $O S$ overall survival, $C S S$ cancer-specific survival, $P O D$ postoperative day, $I Q R$ interquartile range, $m G P S$ modified Glasgow Prognostic Score, poGPS postoperative Glasgow Prognostic Score, $M C V$ mean corpuscular volume

${ }^{\mathrm{a}}$ Males $=\mathrm{Hb}<130 \mathrm{~g} / \mathrm{L}$, females $=<120 \mathrm{~g} / \mathrm{L}$; microcytic $=$ anemia and MCV $<80 \mathrm{fL}$; normocytic $=$ anemia and MCV 80-100 fL

${ }^{\mathrm{b}} 0=\mathrm{CRP}<10 \mathrm{mg} / \mathrm{L} ; 1=\mathrm{CRP} \geq 10 \mathrm{mg} / \mathrm{L}$ and albumin $\geq 35 \mathrm{~g} / \mathrm{L} ; 2=\mathrm{CRP} \geq 10 \mathrm{mg} / \mathrm{L}$ and albumin $<35 \mathrm{~g} / \mathrm{L}$ )

${ }^{\mathrm{c}} 0=\mathrm{CRP}<150 \mathrm{mg} / \mathrm{L} ; 1=\mathrm{CRP} \geq 150 \mathrm{mg} / \mathrm{L}$ and albumin $\geq 25 \mathrm{~g} / \mathrm{L} ; 2=\mathrm{CRP} \geq 150 \mathrm{mg} / \mathrm{L}$ and albumin $<25 \mathrm{~g} / \mathrm{L}$

TABLE 3 Sensitivity analysis: linear regression coefficients of postoperative day $3 \mathrm{C}$-reactive protein concentrations, odds ratios of postoperative complications, and hazard ratios of all-cause mortality with respect to perioperative blood transfusion across the propensity score methods

\begin{tabular}{lllll}
\hline Model & $n$ & POD 3 CRP [B (95\% CI)] & Complication [OR (95\% CI)] & Overall survival [HR (95\% CI)] \\
\hline Unadjusted & 544 & $34(15-54)$ & $3.28(2.03-5.29)$ & $3.18(2.08-4.84)$ \\
Excluding CD grade 3-5 complications & 484 & $28(6-51)$ & - & $2.76(1.65-4.66)$ \\
Propensity score regression & 423 & $31(8-54)$ & $2.07(1.14-3.77)$ & $1.88(1.04-3.41)$ \\
Propensity score matched & 116 & $34(3-64)$ & $2.91(1.36-6.20)$ & $2.38(0.99-5.73)$ \\
\hline
\end{tabular}

$B$ linear regression coefficient, $P O D$ postoperative day, $C D$ Clavien-Dindo, $C R P$ C-reactive protein, $O R$ odds ratio, $H R$ hazard ratio, $C I$ confidence interval

found no independent impact of blood transfusion on colorectal cancer recurrence when corrected for preoperative anemia. ${ }^{28}$ It is unclear why more recent studies tend to report no impact on survival. It may be that modern blood donation, processing, and transfusion guidelines lead to a lower negative risk profile, or it may relate to the analyses used by the reporting groups. With this in mind, the present study included preoperative anemia as a matching variable along with a wider variety of other perioperative variables, and the results are in favor of a negative impact on both short- and long-term outcomes. Given the association between perioperative blood transfusion and postoperative systemic inflammatory response, it is possible that the contribution of blood transfusion to postoperative immunologic dissonance is the mechanism by which such outcomes are affected. ${ }^{29}$ Indeed, this would be in keeping with older evidence reporting even poorer outcomes in patients who both received a perioperative blood transfusion and experienced a postoperative infective complication. ${ }^{30,31}$

In the propensity score matched cohort, perioperative blood transfusion was not associated with sustained significant differences in postoperative $\mathrm{Hb}$. This might suggest that despite the transfusion of PRCs achieving the aim of restoring the oxygen-carrying capacity of the blood, the impact on the host immune system still leads to poorer outcome.

Alternatively, it is possible that more advanced and bulky tumors lead to longer and more difficult surgery, generating a greater inflammatory response, while also being associated with an increased transfusion requirement and a greater likelihood of morbidity and mortality. Although the propensity score matching process in the present study aimed to correct for most of these confounders, its retrospective nature prevents us from making any causal inference between transfusion and postoperative inflammatory response.

The present study has a number of limitations. Its retrospective nature leads to missing data, and the use of propensity score matching, although designed to reduce confounding, can introduce bias in parameters that have not been recorded or are unknown. Furthermore, its use leads to loss of statistical power. The lack of a formal blood transfusion protocol during the study period means that some patients with preoperative anemia did not receive a perioperative blood transfusion, whereas some patients who were not anemic did, presumably due to intraoperative blood loss or other factors. Compounding this, the small numbers of transfused patients prevented meaningful 
TABLE 4 Factors associated with postoperative complications and overall survival in patients who received a perioperative blood transfusion

\begin{tabular}{|c|c|c|c|c|}
\hline Postoperative complications variable & Univariate OR $(95 \% \mathrm{CI})$ & $p$ value & Multivariate OR (95\% CI) & $p$ value \\
\hline Age & $0.85(0.50-1.46)$ & 0.557 & - & - \\
\hline Male sex & $1.29(0.53-3.09)$ & 0.575 & - & - \\
\hline BMI & $0.85(0.53-1.35)$ & 0.483 & - & - \\
\hline ASA & $1.02(0.61-1.72)$ & 0.928 & - & - \\
\hline Laparoscopic & $0.70(0.26-1.84)$ & 0.468 & - & - \\
\hline Dexamethasone & $0.89(0.34-2.32)$ & 0.806 & - & - \\
\hline Rectal & $3.02(1.00-9.31)$ & 0.050 & - & 0.309 \\
\hline $\mathrm{T}$ stage & $0.90(0.56-1.45)$ & 0.660 & - & - \\
\hline $\mathrm{mGPS}^{\mathrm{b}}$ & $0.95(0.57-1.61)$ & 0.853 & - & - \\
\hline poGPS $3^{\mathrm{c}}$ & $2.16(1.24-3.76)$ & 0.007 & $2.47(1.93-3.15)$ & $<0.001$ \\
\hline Overall survival variable & Univariate HR (95\% CI) & $p$ value & Multivariate HR (95\% CI) & $p$ value \\
\hline Age & $1.39(0.85-2.25)$ & 0.182 & - & - \\
\hline Male sex & $1.41(0.66-3.02)$ & 0.373 & - & - \\
\hline BMI & $0.80(0.54-1.18)$ & 0.251 & - & - \\
\hline ASA & $2.19(1.40-3.42)$ & 0.001 & $1.96(1.16-3.33)$ & 0.012 \\
\hline Laparoscopic & $0.44(0.15-1.26)$ & 0.125 & - & - \\
\hline Dexamethasone & $0.68(0.28-1.61)$ & 0.378 & - & - \\
\hline Rectal & $0.64(0.24-1.70)$ & 0.375 & - & - \\
\hline $\mathrm{T}$ stage & $1.89(1.07-3.36)$ & 0.029 & - & 0.165 \\
\hline $\mathrm{mGPS}^{\mathrm{b}}$ & $1.83(1.18-2.84)$ & 0.007 & $1.69(1.08-2.62)$ & 0.020 \\
\hline poGPS $3^{c}$ & $1.39(0.92-2.12)$ & 0.121 & - & - \\
\hline Complication & $1.75(0.77-4.01)$ & 0.183 & - & - \\
\hline
\end{tabular}

ASA American Society of Anesthesiologists grade, BMI body mass index, $C I$ confidence interval, $H R$ hazard ratio, $O S$ overall survival, $m G P S$ modified Glasgow Prognostic Score

${ }^{\mathrm{a}}$ Males $=\mathrm{Hb}<130 \mathrm{~g} / \mathrm{L}$, females $=<120 \mathrm{~g} / \mathrm{L}$

${ }^{\mathrm{b}} 0=\mathrm{CRP}<10 \mathrm{mg} / \mathrm{L} ; 1=\mathrm{CRP} \geq 10 \mathrm{mg} / \mathrm{L}$ and albumin $\geq 35 \mathrm{~g} / \mathrm{L} ; 2=\mathrm{CRP} \geq 10 \mathrm{mg} / \mathrm{L}$ and albumin $<35 \mathrm{~g} / \mathrm{L}$

${ }^{\mathrm{c}} 0=\mathrm{CRP}<150 \mathrm{mg} / \mathrm{L} ; 1=\mathrm{CRP} \geq 150 \mathrm{mg} / \mathrm{L}$ and albumin $\geq 25 \mathrm{~g} / \mathrm{L} ; 2=\mathrm{CRP} \geq 150 \mathrm{mg} / \mathrm{L}$ and albumin $<25 \mathrm{~g} / \mathrm{L}$

subgroup analysis of transfusion timing. Estimated blood loss was recorded very poorly, preventing this important variable from being included in the analysis. Despite this, the similar effect sizes found at sensitivity analysis suggest internal validity to the findings.

\section{CONCLUSIONS}

The present study reports a significant association between perioperative blood transfusion, the postoperative systemic inflammatory response, complications and survival following surgery for colorectal cancer. Furthermore, in those who received a blood transfusion, an exaggerated perioperative innate systemic inflammatory response appeared to compound the negative effects on short- and long-term outcomes. Although the described relationship between blood transfusion and poorer outcomes is not new, the present study suggests a possible mechanism, i.e. modulation of the postoperative systemic inflammatory response.

ACKNOWLEDGMENTS The authors thank Amanda Stewart, Scottish National Blood Chain Analytics, and Moira Caldwell, Transfusion Practitioner, NHS Greater Glasgow and Clyde, for obtaining data regarding blood transfusions.

DISCLOSURE Stephen T. McSorley, Alexander Tham, Ross D. Dolan, Colin W. Steele, Jason Ramsingh, Campbell Roxburgh, Paul G. Horgan, and Donald C. McMillan have no conflicts of interest to declare.

OPEN ACCESS This article is distributed under the terms of the Creative Commons Attribution 4.0 International License (http://crea tivecommons.org/licenses/by/4.0/), which permits unrestricted use, distribution, and reproduction in any medium, provided you give appropriate credit to the original author(s) and the source, provide a link to the Creative Commons license, and indicate if changes were made. 


\section{REFERENCES}

1. Acheson AG, Brookes MJ, Spahn DR. Effects of allogeneic red blood cell transfusions on clinical outcomes in patients undergoing colorectal cancer surgery. Ann Surg. 2012;256:235-244.

2. McDermott FD, Heeney A, Kelly ME, et al. Systematic review of preoperative, intraoperative and postoperative risk factors for colorectal anastomotic leaks. Br J Surg. 2015;102:462-479

3. Pang QY, An R, Liu HL. Perioperative transfusion and the prognosis of colorectal cancer surgery: a systematic review and meta-analysis. World J Surg Oncol. 2019;17(1):7.

4. Amato A, Pescatori M. Perioperative blood transfusions for the recurrence of colorectal cancer. Cochrane Database Syst Rev. 2006;(1):CD005033

5. Mynster T, Christensen IJ, Moesgaard F, et al. for the Danish RANX05 Colorectal Cancer Study Group. Effects of the combination of blood transfusion and postoperative infectious complications on prognosis after surgery for colorectal cancer. $\mathrm{Br}$ J Surg. 2000;87:1553-1562

6. Blumberg N, Heal JM. Effects of transfusion on immune function. Cancer recurrence and infection. Arch Pathol Lab Med. 1994;118(4):371-379

7. Watt DG, McSorley ST, Park JH, Horgan PG, McMillan DC. A postoperative systemic inflammation score predicts short- and long-term outcomes in patients undergoing surgery for colorectal cancer. Ann Surg Oncol. 2017;24(4):1100-1109

8. McSorley ST, Roxburgh CSD, Horgan PG, McMillan DC. The impact of preoperative dexamethasone on the magnitude of the postoperative systemic inflammatory response and complications following surgery for colorectal cancer. Ann Surg Oncol. 2017;24(8):2104-2112

9. Sandini M, Ruscic KJ, Ferrone CR, Warshaw AL, Qadan M, Eikermann $\mathrm{M}$, et al. Intraoperative dexamethasone decreases infectious complications after pancreaticoduodenectomy and is associated with long-term survival in pancreatic cancer. Ann Surg Oncol. 2018;25(13):4020-4026

10. Roxburgh CS, Horgan PG, McMillan DC. The perioperative immune/inflammatory insult in cancer surgery: time for intervention? OncoImmunology 2013;2:e27324

11. Watt DG, Horgan PG, McMillan DC. Routine clinical markers of the magnitude of the systemic inflammatory response after elective operation: a systematic review. Surgery 2015; 157:362-80

12. McSorley ST, Dolan RD, Roxburgh CS, Horgan PG, MacKay GJ, McMillan DC. Possible dose dependent effect of perioperative dexamethasone and laparoscopic surgery on the postoperative systemic inflammatory response and complications following surgery for colon cancer. Eur J Surg Oncol. 2019;45(9):1613-1618.

13. Quinn EM, Meland E, McGinn S, Anderson JH. Correction of iron-deficiency anaemia in colorectal surgery reduces perioperative transfusion rates: a before and after study. Int $J$ Surg. 2017;38:1-8

14. Watt DG, Ramanathan ML, McSorley ST, Walley K, Park JH, Horgan PG, et al. Clinicopathological determinants of an elevated systemic inflammatory response following elective potentially curative resection for colorectal cancer. Ann Surg Oncol. 2017;24(9):2588-2594

15. World Health Organization. Haemoglobin concentrations for the diagnosis of anaemia and assessment of severity. Vitamin and Mineral Information System. Geneva: World Health Organization; 2011 (WHO/NMH/NHD/MNM/11.1)
16. McMillan DC. The systemic inflammation based Glasgow Prognostic Score: a decade of experience in patients with cancer. Cancer Treat Rev. 2013;39(5):534-540

17. Dindo D, Demartines N, Clavien PA. Classification of surgical complications: a new proposal with evaluation in cohort of 6336 patients and results of a survey. Ann Surg. 2004;240(2):205-213

18. Platt JJ, Ramanathan ML, Crosbie RA, et al. C-reactive protein as a predictor of postoperative infective complications after curative resection in patients with colorectal cancer. Ann Surg Oncol. 2012;19:4168-4177

19. Rhode JM, Dimcheff DE, Blumberg N, Saint S, Langa KM, Kuhn $\mathrm{L}$, et al. Health care-associated infection after red blood cell transfusion: a systematic review and meta-analysis. JAMA 2014;311(13):1317-1326

20. Matsuda S, Takeuchi H, Kawakubo H, Fukuda K, Nakamura R, Takahashi $\mathrm{T}$, et al. Correlation between intense postoperative inflammatory response and survival of esophageal cancer patients who underwent transthoracic esophagectomy. Ann Surg Oncol. 2015;22(13):4453-4460

21. Saito T, Kurokawa Y, Miyazaki Y, Makino T, Takahashi T, Yamasaki M, et al. Which is a more reliable indicator of survival after gastric cancer surgery: postoperative complication occurrence or C-reactive protein elevation? J Surg Oncol. 2015;112(8):894-899

22. McSorley ST, Watt DG, Horgan PG, McMillan DC. Postoperative systemic inflammatory response, complication severity, and survival following surgery for colorectal cancer. Ann Surg Oncol. 2016;23(9):2832-2840

23. Hensler T, Hecker H, Heeg K, Heidecke CD, Bartels H, Barthlen W, et al. Distinct mechanisms of immunosuppression as a consequence of major surgery. Infect Immun. 1997;65:2283-2291

24. Ruffell B, Coussens LM. Macrophages and therapeutic resistance in cancer. Cancer Cell. 2015;27(4):462-72.

25. Wu HL, Tai YH, Lin SP, Chan MY, Chen HH, Chang KY. The impact of blood transfusion on recurrence and mortality following colorectal cancer resection: a propensity score analysis of 4030 patients. Sci Rep. 2018;8:13345

26. Tarantino I, Ukegjini K, Warschkow R, et al. Blood transfusion does not adversely affect survival after elective colon cancer resection: a propensity score analysis. Langenbecks Arch Surg. 2013;398:841-849

27. Warschkow R, Guller U, Koberle D, et al. Perioperative blood transfusions do not impact overall and disease-free survival after curative rectal cancer resection. Ann Surg. 2014;259(1):131-138

28. Morner ME, Edgren G, Martling A, Gunnarsson U, Egenvall M. Preoperative anaemia and perioperative red blood cell transfusion as prognostic factors for recurrence and mortality in colorectal cancer-a Swedish cohort study. Int $J$ Colorectal Dis. 2017;32(2):223-232.

29. Goldfarb Y, Sorksi L, Benish M, Levi B, Melamed R, BenEliyahu S. Improving postoperative immune status and resistance to cancer metastasis: a combined perioperative approach of immunostimulation and prevention of excessive surgical stress response. Ann Surg. 2011;253:798-810

30. Tartter PI. Blood transfusion and infectious complications following colorectal cancer surgery. Br J Surg. 1988;75(8):789-792

31. Tartter PI. Blood transfusion and post-operative infections. Transfusion 1989;299(5):456-9

Publisher's Note Springer Nature remains neutral with regard to jurisdictional claims in published maps and institutional affiliations. 